Original article

\title{
Adolescent psychosis risk symptoms predicting persistent psychiatric service use: A 7-year follow-up study
}

\author{
Maija Lindgren $^{\mathrm{a}, *}$, Minna Jonninen $^{\mathrm{b}}$, Markus Jokela $^{\mathrm{b}}$, Sebastian Therman ${ }^{\mathrm{a}}$ \\ ${ }^{a}$ Mental Health Unit, National Institute for Health and Welfare, Helsinki, Finland \\ ${ }^{\mathrm{b}}$ Department of Psychology and Logopedics, Faculty of Medicine, Helsinki University, Helsinki, Finland
}

\section{A R T I C L E I N F O}

\section{Article history:}

Received 17 July 2018

Received in revised form 15 October 2018

Accepted 16 October 2018

Available online 15 November 2018

\section{Keywords:}

Psychiatry

Prodromal questionnaire

Help-seeking

Service use

Trajectory

\begin{abstract}
A B S T R A C T
Background: We investigated whether psychosis risk symptoms predicted psychiatric service use using seven-year register follow-up data.

Methods: Our sample included 715 adolescents aged 15-18, referred to psychiatric care for the first time. Psychosis risk symptoms were assessed with the Prodromal Questionnaire (PQ) at the beginning of the treatment. We assessed the power of the overall PQ as well as its positive, negative, general, and disorganized psychosis risk symptom factors in predicting prolonged service use. Baseline psychiatric diagnoses (grouped into 7 categories) were controlled for. Based on both inpatient and outpatient psychiatric treatment after baseline, adolescents were divided into three groups of brief, intermittent, and persistent service use.

Results: Stronger symptoms on any PQ factor as well as the presence of a mood disorder predicted prolonged service use. All of the PQ factors remained significant predictors when adjusted for baseline mood disorder and multimorbidity.

Conclusions: In a prospective follow-up of a large sample using comprehensive mental health records, our findings indicate that assessing psychosis risk symptoms in clinical adolescent settings at the beginning of treatment could predict long-term need for care beyond diagnostic information. Our findings replicate the previous findings that positive psychosis risk symptoms are unspecific markers of severity of psychopathology. Also psychosis risk symptoms of the negative, disorganization, and general clusters are approximately as strongly associated with prolonged psychiatric service use in the upcoming years.
\end{abstract}

(C) 2018 Elsevier Masson SAS. All rights reserved.

\section{Introduction}

Positive psychotic-like symptoms, such as perceptual abnormalities and delusional ideas, are rather common and usually transient in the general population. By definition, these psychoticlike symptoms are subclinical, as in that they do not reach the psychotic threshold, and differ from the positive symptoms of a frank psychotic disorder quantitatively. However, psychotic-like symptoms may be a developmental expression of proneness to psychosis, which due to environmental adversity and other risk factors interacting with a genetic risk can become more persistent and lead to a clinically relevant disorder [1]. For that reason, psychotic-like symptoms can be referred to as "positive psychosis risk symptoms", which term is used hereinafter in this paper. As well as psychotic disorders [2], positive psychosis risk symptoms

\footnotetext{
* Corresponding author at: Mental Health Unit, National Institute for Health and Welfare (THL), PO Box 30, FIN-00271 Helsinki, Finland.

E-mail address: maija.lindgren@thl.fi (M. Lindgren).
}

may indicate a vulnerability to a wider spectrum of psychopathology $[3,4]$. Positive psychosis risk symptoms co-occur with a varied form of psychopathology and are not confined to any one psychiatric disorder. In addition, those reporting positive psychosis risk symptoms more often suffer from multiple disorders, and concurrent positive symptoms are associated with disorder severity, distress, and poorer prognosis [2,5-7]. In the general population positive psychosis risk symptoms are associated with help-seeking and unmet need for care [8-12] and later psychiatric hospital treatments for severe mental disorders [13]. A recent review reported that in the general population, people with positive psychosis risk symptoms were twice as likely to report mental health service use compared to those without these symptoms, but service use was almost always assessed retrospectively and only by self-report [14].

In addition to positive psychosis risk symptoms, other psychosis risk symptoms include negative, disorganization, and general risk symptoms. Positive symptoms [15] as well as negative symptoms [16] are associated with heightened risk for psychotic disorders. As to other outcomes besides psychotic disorder, negative and disorganization 
symptoms predict poor functioning during follow-up among at-risk individuals [17]. In one study, non-specific subjective anomalies in thinking and attention were more relevant to help-seeking and needfor-care than positive symptoms [18].

Adolescents referred to psychiatric care for the first time have heterogeneous service needs over time and it is not clear how psychosis risk symptoms are associated with help-seeking behavior in adolescent psychiatric populations. The current study aims to investigate whether psychosis risk symptoms - as assessed with the Prodromal Questionnaire (PQ) - predict further patterns of treatment of adolescent non-psychotic patients. It was hypothesized that co-occurring psychosis risk symptoms at entry would be associated with prolonged subsequent mental health service use, making the PQ a potentially useful tool for screening for not just the risk for psychosis but also the risk for long-term service use. We also wanted to investigate whether distinct factors of psychosis risk symptoms correlate with service use differently. As adolescents with long-term need for help form a group warranting special attention, identifying factors associated with persistent service use may help to develop early prevention and intervention strategies.

\section{Methods}

\subsection{Participants and study protocol}

We utilized the sample of the Helsinki Prodromal Study, a prospective study investigating psychosis risk [19]. All new patients aged 15-18 years entering adolescent psychiatric inpatient or outpatient clinic or ward in Helsinki, Finland were invited to participate in the study during years 2003-2004 and 20072008, the enrolment phase constituting a three year period. The adolescents were asked to fill out the PQ [20] on their first or second visit to the unit. 819 questionnaires were returned, totaling up to $75 \%$ of the eligible participants in psychiatric treatment. The patients who returned the PQ form did not differ from the other patients with regards to age $(t(1611.2)=-0.304, p=.761)$ or gender $\left(\mathrm{X}^{2}=1.920, \mathrm{df}=1, \mathrm{p}=.166\right)$.

Adolescents who were diagnosed with a psychotic disorder at the time of or before the administration of the PQ $(n=27)$ or those who forbid the use of follow-up register data $(n=61)$ were excluded from the analyses. The PQ factor solution was made with these 731 adolescents. Finally we excluded 16 participants who according to the psychiatric register were diagnosed with psychotic disorders at baseline (ICD-10 codes F20, F22-F29, F30.2, F31.2, F31.5, F32.3, or F33.3), resulting in a final sample of 715 adolescents. The mean age of the final sample was 16.5 years and 489 (68.4\%) were female. 27 participants (3.8\%) completed the $\mathrm{PQ}$ in a psychiatric hospital and the rest were outpatients.

The review boards of the National Institute for Health and Welfare and the Ethics Committee of the Hospital District of Helsinki and Uusimaa reviewed and approved the study procedure, the PQ being part of the standard treatment of the adolescents entering psychiatric units.

\subsection{Prodromal questionnaire}

Psychosis risk symptoms were assessed using the Finnish version of the PQ a 92-item self-report survey assessing psychosis risk symptoms with a Yes/No response format [20,21]. For use in later regression modeling, we extracted maximum a posteriori factor scores from two confirmatory factor analysis (CFA) models: 1) the four a priori subfactors (positive, negative, disorganized, and general symptoms), and 2) the full PQ as a single dimension. These item factor analyses used the WLSMV algorithm, and took the dichotomous nature of the items into account. Factor model fit was quantified with the root mean square error of approximation
(RMSEA), the comparative fit index (CFI), and explained common variance (ECV), and was adequate both for the single-dimensional (RMSEA 0.042, CFI 0.87, ECV 36\%) and the four-dimensional models (RMSEA 0.040, CFI 0.88, ECV 40\%). Item factor loadings and thresholds are presented in Supplementary Table 1 and subfactor correlations in Supplementary Table 2.

\subsection{Psychiatric register data}

Baseline diagnoses of the adolescents were acquired from the Finnish hospital discharge database, a part of the Care Registration for Health Care (HILMO), with diagnoses between 30 days before and 30 days after the date of the PQ defined here as the baseline diagnosis. Psychiatric baseline diagnoses were grouped as non-psychotic mood disorders (ICD-10 codes F3X); anxiety disorders (F4X); eating disorders (F5X); substance use disorders (F1X); intentional selfharm (X69-X84, Y87, Y87.0, Z91.5, or Z72.8); disorders usually first diagnosed in infancy, childhood, or adolescence (F6X-F9X, excluding F60.X and F99); and other psychiatric disorders (other F class diagnoses), to be used as covariates in the regression models predicting service use. A person could have a baseline diagnosis from several of these categories.

Follow-up data on the use of psychiatric services was available until the end of the year 2015 including psychiatric treatment in any public outpatient or inpatient clinic or ward. As the last participants were enrolled in year 2008, a seven-year follow up was available for them. The same length of follow-up was used for all participants, constituting data for the subsequent seven years after the completion of the PQ for each adolescent. The first followup year for each participant was 365 days starting the day after the completion of the $P Q$, the second year 365 days after that and so on. For each follow-up year, service use was coded as a dichotomous indicator $(0=$ no treatment during the year, $1=$ treatment during the year). Based on the 7 repeated measurements, we formed three groups as follows: Brief contact: no service use after the first two years; Persistent contact: service use on five or more years during the seven-year follow up; and Intermittent contact: adolescents who did not fall into either one of the other categories.

\subsection{Data analyses}

Factor analyses were conducted with Mplus 8.0 [22] and other analyses with IBM SPSS Statistics for Windows, version 25. Multinomial logistic regressions were conducted to test which variables predicted long-term service use, using the Brief contact group as the reference group. First, we tested which background and clinical variables were the individually significant predictors at a $\mathrm{p}<.05$ level (gender, age, inpatient status, baseline diagnosis categories, multimorbidity, and the PQ symptom factor scores).

To test whether psychosis risk symptoms would predict psychiatric service use beyond diagnostic information, the significant background predictors were included in the final regression models in addition to the PQ factors. The predictiveness of self-reported psychosis risk symptoms was investigated in five separate models (one for each of the four PQ subfactors and the overall factor) because they were very highly correlated with each other, and multicollinearity concerns precluded their use in the same model.

Odds ratios (OR) and their 95\% confidence intervals (CI) were used to estimate the effects; for continuous variables (PQ factors) OR values are given per standard deviation unit change.

\section{Results}

The baseline diagnosis groups of the adolescents can be seen in Table 1 , the most common diagnoses falling into the mood disorder 
category (29.2\%). 237 adolescents (33.1\%) had not been given any diagnosis between 30 days before and after filling out the PQ. The rest had at least one baseline diagnosis; 423 (59.2\%) from one category, 47 (6.6\%) from two categories, and 8 (1.1\%) from 3 or 4 categories. For further analyses, the adolescents were dichotomized in 0-1 diagnostic categories versus $2-4$ diagnostic categories (multimorbidity).

The rates of psychiatric treatment over the follow-up time can be seen in Fig. 1. Patterns of long-term trajectories were identified based on psychiatric service use during the seven first years after the first psychiatric visit. Of the adolescents, 311 (43.5\%) were classified to the Brief contact group, 219 (30.6\%) to the Intermittent contact group, and 185 (25.9\%) to the Persistent contact group (Table 1). All baseline PQ scores were the highest in the Persistent group and lowest in the Brief group (Fig. 2).

As described in the Methods, diagnosable psychotic disorders at baseline were excluded from the sample. Although transition to psychosis was not the focus of this study, of note, 67 emerging psychosis cases (40 females and 27 males; 9.4\% of the whole sample) were identified during the 7-year follow-up from the hospital discharge register. These individuals most often used psychiatric services persistently (76.1\%) or intermittently (19.4\%) during the follow-up phase.

The predictiveness of each background variable with regards to service use trajectory was investigated with separate multinomial logistic regression models (Supplementary Table 3). Baseline inpatient status, age, or gender did not predict prolonged service use and were therefore not included as covariates in further analyses. Of the baseline diagnosis categories, mood disorders were significantly associated with persistent service use, as was multimorbidity, and these variables were therefore included in the final models as covariates.

Table 2 shows the parameters of the final models for the four domains of psychosis risk symptom factors and the overall factor. All PQ factors were statistically significant predictors of prolonged (intermittent and persistent) service use, and the differences between the models were small.

Psychosis during follow-up predicted long-term service use (Supplementary Table 3). To see whether the association between baseline psychosis risk symptoms and long-term service use was explained by the subgroup of participants developing psychosis during the follow-up, we repeated the final regression analyses excluding the participants with follow-up psychosis. The results did not change (Supplementary Table 4): all four clusters of psychosis risk symptoms still significantly predicted service use trajectory.

\section{Discussion}

We wanted to assess whether different types of self-reported psychosis risk symptoms would predict psychiatric service use over the follow-up period of seven years. We investigated nonpsychotic adolescents who had been referred to psychiatric care for the first time, using register data of their long-term psychiatric treatment service use. Different psychiatric service needs could be identified in subgroups of adolescents, $44 \%$ of the adolescents using services quite briefly, 31\% intermittently, and 26\% persistently along the seven years of follow-up. The predictive power of psychosis risk symptoms in early or mid-adolescence over later years in adulthood was examined, as the adolescents were followed up until age $22-25$. Correlates of more persistent service use were mood disorders and diagnosis from multiple categories at baseline, as well as negative, general, disorganization, and positive psychosis risk symptoms at the beginning of treatment. Psychosis risk symptoms predicted service use even when developing psychosis during follow-up was accounted for.

Genders did not differ in service use trajectories. A previous study found gender to play a significant role in seeking help for mental health problems [23] but it has to be noted that in the current study, over $2 / 3$ of the participants were female. Furthermore, age was not associated with service use, which might be due to the small variation in age. Inpatient status at baseline also did not predict service use, perhaps due to the small amount of PQs filled out by inpatients.

Mood disorders at baseline were associated with persistent service use. Mood and anxiety symptoms often occur together with positive psychosis risk symptoms and may represent a prodrome of psychosis in some cases, but most individuals presenting with both symptom clusters will not develop psychosis. Previous literature has reported depressive symptoms to contribute to positive psychosis risk symptoms in the general population, suggesting shared vulnerability for affective dysregulation and

Table 1

Participants by psychiatric service use trajectory. Frequency (\%) or range, mean (standard deviation).

\begin{tabular}{|c|c|c|c|c|c|}
\hline & \multirow[t]{2}{*}{ Total, $\mathrm{n}=715$} & \multicolumn{3}{|l|}{ Service use group } & \multirow[t]{2}{*}{ Group difference } \\
\hline & & Brief, $n=311(43.5 \%)$ & Intermittent, $\mathrm{n}=219(30.6 \%)$ & Persistent, $\mathrm{n}=185$ (25.9\%) & \\
\hline Age & $\begin{array}{l}15.0-18.9 \\
16.5(0.9)\end{array}$ & $\begin{array}{l}15.0-18.5 \\
16.5(0.9)\end{array}$ & $\begin{array}{l}15.0-18.9 \\
16.4(0.9)\end{array}$ & $\begin{array}{l}15.0-18.1 \\
16.5(0.9)\end{array}$ & ns \\
\hline Females & $489(68.4 \%)$ & $200(64.3 \%)$ & $156(71.2 \%)$ & $133(71.9 \%)$ & ns \\
\hline Inpatient at baseline & $27(3.8 \%)$ & $9(2.9 \%)$ & $9(4.1 \%)$ & $9(4.9 \%)$ & ns \\
\hline PQ total score & $0-86,31.0(18.6)$ & $0-77,26.0(17.2)$ & $0-77,32.5(18.8)$ & $0-86,37.4(18.6)$ & $\mathbf{p}<. \mathbf{0 0 1}$ \\
\hline \multicolumn{6}{|c|}{ PQ symptom factor raw sum scores } \\
\hline Positive & $0-36,10.5(7.7)$ & $0-32,9.1(7.3)$ & $0-36,10.8(8.1)$ & $0-35,12.5(7.6)$ & $\mathbf{p}<. \mathbf{0 0 1}$ \\
\hline Negative & $0-18,6.4(4.8)$ & $0-17,5.1(4.2)$ & $0-18,7.0(4.9)$ & $0-18,8.1(5.2)$ & $\mathbf{p}<.001$ \\
\hline Disorganization & $0-19,6.9(4.8)$ & $0-17,5.7(4.4)$ & $0-19,7.2(4.8)$ & $0-19,8.6(4.8)$ & $\mathbf{p}<.001$ \\
\hline General & $0-15,7.2(4.1)$ & $0-14,6.2(4.0)$ & $0-15,7.6(4.1)$ & $0-15,8.3(3.8)$ & $\mathbf{p}<. \mathbf{0 0 1}$ \\
\hline \multicolumn{6}{|c|}{ Baseline diagnosis category } \\
\hline Mood & $209(29.2 \%)$ & $75(24.1 \%)$ & $68(31.1 \%)$ & $66(35.7 \%)$ & $p=.026$ \\
\hline Anxiety & $154(21.5 \%)$ & $62(19.9 \%)$ & $41(18.7 \%)$ & $51(27.6 \%)$ & ns \\
\hline Eating & $52(7.3 \%)$ & $19(6.1 \%)$ & $15(6.8 \%)$ & $18(9.7 \%)$ & ns \\
\hline Substance & $17(2.4 \%)$ & $9(2.9 \%)$ & $5(2.3 \%)$ & $3(1.6 \%)$ & ns \\
\hline Childhood/adolesc. & $94(13.1 \%)$ & $39(12.5 \%)$ & $28(12.8 \%)$ & $27(14.6 \%)$ & ns \\
\hline Self-harm & $7(1.0 \%)$ & $4(1.3 \%)$ & $3(1.4 \%)$ & 0 & ns \\
\hline Other psychiatric & $10(1.4 \%)$ & $3(1.0 \%)$ & $5(2.3 \%)$ & $2(1.1 \%)$ & ns \\
\hline
\end{tabular}

$\mathrm{PQ}$, Prodromal Questionnaire.

ns, nonsignificant.

a overall group difference, Kruskal-Wallis test or Chi-square test. 


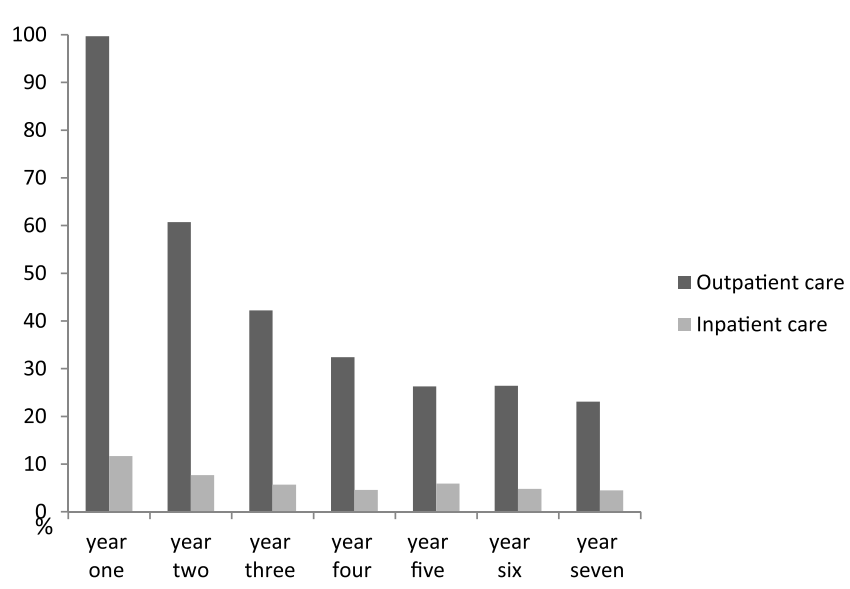

Fig. 1. Rates of psychiatric treatment over the seven-year follow-up, $n=715$.

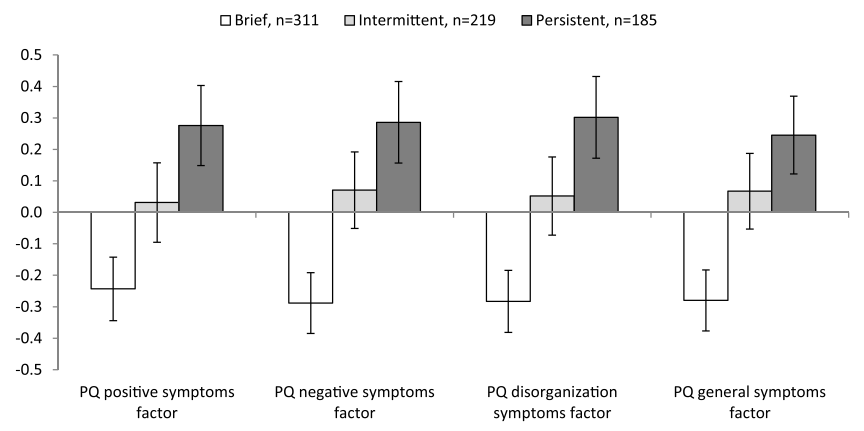

Fig. 2. Prodromal Questionnaire standardized factor score means and $95 \%$ confidence intervals among the three service use trajectory groups, $n=715$.

Table 2

Predicting the service use trajectories with PQ factors with Brief service use as the reference category, $n=715$. Separate regression models for each PQ factor. Baseline mood disorder and multimorbidity have been controlled for in every model.

\begin{tabular}{lll}
\hline & \multicolumn{2}{l}{ Predicted trajectory, OR $(95 \% \mathrm{CI})$} \\
\cline { 2 - 3 } Standardized PQ symptom factor & Intermittent & Persistent \\
\hline Single factor (all 94 items) & $1.5(1.2,1.8), \mathrm{p}<.001$ & $1.9(1.6,2.4), \mathrm{p}<.001$ \\
Positive symptoms & $1.4(1.1,1.7), \mathrm{p}=.001$ & $1.8(1.5,2.3), \mathrm{p}<.001$ \\
Negative symptoms & $1.6(1.3,1.9), \mathrm{p}<.001$ & $2.1(1.6,2.6), \mathrm{p}<.001$ \\
Disorganization symptoms & $1.5(1.2,1.8), \mathrm{p}<.001$ & $2.0(1.6,2.6), \mathrm{p}<.001$ \\
General symptoms & $1.6(1.3,1.9), \mathrm{p}<.001$ & $2.0(1.6,2.5), \mathrm{p}<.001$ \\
\hline
\end{tabular}

PQ, Prodromal Questionnaire.

OR, Odds Ratio per unit change; $95 \% \mathrm{CI}, 95 \%$ Confidence Interval.

reality distortion [24]. In psychosis risk individuals, comorbid depressive and anxiety disorders are common and are associated with lower functioning and worse longitudinal outcome [25]. Cooccurrence of affective and positive symptoms is associated with worse outcome in terms of risk of developing psychosis [26] and poorer outcome of depressive disorder [27]. In the current study, there was no substantial change in predictiveness when the presence of mood disorders was controlled for, indicating that the predictive power of positive psychotic symptoms did not depend on concurrent mood problems. In addition, psychosis risk symptoms continued to predict service use also when multimorbidity at baseline was controlled for.

Our results indicate that it might be possible to distinguish patients with longer need for care by assessing psychosis risk symptoms at initial psychiatric help-seeking. Our results are congruent with earlier studies linking positive symptoms of young people with poorer illness course and greater service use [7]. In adolescents with non-psychotic psychiatric disorders, those with co-occurring positive psychosis risk symptoms showed nonadaptive coping and poorer functioning compared to patients without positive symptoms [28]. Bhavsar and colleagues [9] similarly linked data of subclinical psychotic symptoms with prospective health records, finding that they were related to subsequent mental healthcare need and longer treatment in the general population. Among psychiatric outpatients, positive psychosis risk symptoms were associated with higher levels of symptomatology across various diagnostic domains indicating general clinical severity [29]. In a youth cohort, those with psychosis risk symptoms at baseline but not at 2-year follow-up nonetheless scored higher on symptoms and lower on functioning at follow-up, as compared to youths without baseline psychosis risk symptoms [30]. In the adult population, positive psychosis risk symptoms increased the risk for psychiatric hospitalization in a dose-response manner [13], the probability of help-seeking increasing as a function of multiple positive symptoms [11]. Positive risk symptoms may thus be complicating factors in nonpsychotic illness, associated with more severe psychopathology [7]. These findings indicate how positive psychosis risk symptoms may be unspecific indicators of general mental health and the severity of the challenges the person is facing. In line with this, it has been suggested that positive symptoms may be a marker of the more severe end of a common mental distress continuum in teenagers [31].

The PQ not only includes positive psychotic symptoms but also negative, disorganized, and general psychosis risk symptoms. Of note, not only positive risk symptoms were predictive of more persistent service use, but negative, general and disorganized risk symptoms were equally predictive. The role of negative symptoms as psychosis risk symptoms is somewhat underrepresented [32] as the ultra-high risk and clinical high-risk criteria are based on 
positive symptoms only. However, studies show that the negative symptom cluster also is predictive of psychosis [16] and poorer functioning [17]. Different symptom clusters can be difficult to separate from each other; once one of these symptom clusters exacerbate, so do the others [33-35]. Also in the current sample, the symptom factors were highly correlated with each other. As it seems that positive psychosis risk symptoms often overlap with negative or depressive symptoms, the PQ could be an asset in preliminary assessment of psychiatric patients as it incorporates all of these risk symptom clusters.

The four factor model of psychosis risk symptoms followed the a priori classification of the PQ instrument [20]. It was used instead of others models (e.g. our previous 9-factor solution [36]) to reduce the number of tested variables and enhance clinical usability of the results. Positive psychosis risk symptoms were here investigated as one construct, but subdimensions of positive symptoms may affect psychopathology differently. For instance, persecutory ideation and bizarre experiences may subjectively be more disruptive, whereas magical thinking and grandiosity might not confer distress and be as suggestive of psychopathology as other positive risk symptoms $[37,38]$. In one study in a non-clinical sample, of different positive symptoms, only auditory perceptual disturbances were associated with need for psychological services, when neurotic traits, anxiety, and depressive symptoms were controlled for [39].

Some types of positive psychosis risk symptoms may cause more distress and affect help-seeking more than others. Not all positive symptoms lead to need for care [40], and a distinction can be made between subclinical psychotic symptoms that generate distress and help-seeking and the more common psychotic-like experiences that do not $[1,3]$. The individual's appraisal of the positive symptom is of great importance with regards to associations with psychopathology [41], and the level of distress caused by symptoms is a major factor on whether the person seeks for help [42]. A previous study indicated that magical thinking also is associated with psychopathology if it is accompanied with distress [41], and another study found that hearing voices was the only symptom connected to help-seeking when perceived distress was controlled for [42].

In non-psychotic psychiatric disorders, positive psychosis risk symptoms are common [43]; the impact of positive symptoms and psychiatric disorders being reciprocal, as both may affect the other. Having positive symptoms may increase the distress caused by a psychiatric disorder, or, conversely, having a psychiatric disorder may give rise to psychosis risk symptoms and affect the way they are attributed [44]. There may also be shared vulnerability factors behind the two [45]. As risk symptoms do not necessarily cause distress [3], the person having them may not seek for help; however, having a psychiatric disorder may create or intensify the feelings of distress and lead to help-seeking even though psychosis risk symptoms themselves may not [46]. These findings indicate the importance of distress to help-seeking behavior.

\subsection{Strengths and limitations}

This prospective study utilized a large sample of $15-18$ yearolds who had been referred to psychiatric care for the first time; the results can be generalized to general psychiatric care in the adolescent age group. Previous studies have often relied on selfreported service use [14], vulnerable to recall bias. Objective service use data with the seven-year register follow-up were available in the current study, enabling detecting trajectories in help-seeking behavior. The service use group definitions were made to be easily understood and replicable.

Service need is not the same thing as service use, as some adolescents may have needed help for a longer time but did not continue using the services due to, for instance, unwillingness, inability, or service availability. In other words, longer service use only partly reflects the severity of mental health problems. Another limitation was that we obtained information only from public services - however, private service use is not common among adolescents in Finland.

The information of the participants' baseline diagnoses was available and was used as a covariate, so the association between psychosis risk symptoms and service use was not simply attributable to the influence of concurrent mental health disorders. Due to power considerations, the grouping of the diagnoses was rough and did not take into account the varying severity level of the various diagnoses included in each group. In addition to this diagnosis information, only the PQ was available for the whole sample, and controlling for the possible confounding effects of other concurrent mental health symptoms or level of functioning was not possible.

In the current study, there was no information on how distressing the psychosis risk symptoms were to the participants, as the PQ version with only Yes/No answers was used. Screening effectiveness of the PQ could be improved by including the distress criteria when probing for psychosis risk symptoms [47], as in the PQ-B.

\subsection{Conclusions}

The current study examined how different types of psychosis risk symptoms may predict psychiatric treatment in a seven-year follow-up using a clinical adolescent sample. We wanted to explore whether it is clinically useful to screen for psychosis risk symptoms predicting longer need for care. Identifying different treatment needs and the more harmful psychosis risk symptoms may improve care offered to adolescents seeking help, by targeting otherwise unidentified symptoms for treatment. In the context of psychosis-risk intervention studies, symptom-specific treatment has been shown to decrease symptom levels [48], which is relevant to a much larger patient group than those deemed to be at elevated psychosis risk.

In this sample of adolescents of 15-18 years of age, it was found that psychosis risk symptoms assessed at the beginning of treatment were associated with long-term psychiatric service use. Thus, even though these symptoms are not always indicative of psychopathology - let alone psychosis - they have an association with persistent service use in help-seeking adolescents. Assessing psychosis risk symptoms of adolescents at the beginning of treatment may help to predict their need for care for the upcoming years, and to target interventions.

Attenuated positive symptoms act as a marker of stress and mental suffering [35] and predict psychiatric treatment needs later in life. This study adds to the previous literature in showing that negative, disorganization, and general psychosis risk symptoms may be equally predictive of the severity of mental health challenges as the positive risk symptoms are. Psychosis risk symptoms thus do not only partially predict psychotic and nonpsychotic disorders but also prolonged need for care among psychiatric patients, irrespective of diagnosis category. Assessing all the psychosis risk symptom dimensions of a young person entering psychiatric care is therefore meaningful, regardless of any indications of heightened psychosis risk, as all the dimensions are predictive of long-term need for care.

\section{Funding body agreements and policies}

This study was funded by the Yrjö Jahnsson Foundation (\#6781 to $\mathrm{ML}$ ), the Juho Vainio Foundation (ML), and the Academy of Finland (\#311578 to MJ). The funding organizations played no 
further part in study design, in the collection, analysis and interpretation of data, or in writing of the paper.

\section{Conflict of interest}

ML has received an interview honorarium from Lundbeck. All other authors declare that they have no conflicts of interest.

\section{Acknowledgements}

The authors wish to thank Marjut Grainger and the whole Helsinki Prodromal Study work group, as well as all the participants of the study.

\section{Appendix A. Supplementary data}

Supplementary material related to this article can be found, in
online version, at doi:https://doi.org/10.1016/j.
urpsy.2018.10.004.

\section{References}

[1] van Os J., Linscott RJ, Myin-Germeys I, Delespaul P, Krabbendam L. A systematic review and meta-analysis of the psychosis continuum: evidence for a psychosis proneness-persistence-impairment model of psychotic disorder. Psychol Med 2009;39:179-95, doi:http://dx.doi.org/10.1017/ S0033291708003814.

[2] Fusar-Poli P, Borgwardt S, Bechdolf A, Addington J, Riecher-Rössler A, SchultzeLutter F, et al. The psychosis high-risk state: a comprehensive state-of-the-art review. JAMA Psychiatry 2013;70:107-20, doi:http://dx.doi.org/10.1001/ jamapsychiatry.2013.269.

[3] Linscott RJ, van Os J. An updated and conservative systematic review and metaanalysis of epidemiological evidence on psychotic experiences in children and adults: on the pathway from proneness to persistence to dimensional expression across mental disorders. Psychol Med 2013;43:1133-49, doi:http:// dx.doi.org/10.1017/S0033291712001626.

[4] Kaymaz N, Drukker M, Lieb R, Wittchen HU, Werbeloff N, Weiser M, et al. Do subthreshold psychotic experiences predict clinical outcomes in unselected non-help-seeking population-based samples? A systematic review and metaanalysis, enriched with new results. Psychol Med 2012;42:2239-53, doi: http://dx.doi.org/10.1017/S0033291711002911.

[5] Hanssen M, Peeters F, Krabbendam L, Radstake S, Verdoux H, van Os J. How psychotic are individuals with non-psychotic disorders? Soc Psychiatry Psychiatr Epidemiol 2003;38:149-54, doi:http://dx.doi.org/10.1007/s00127003-0622-7.

[6] Kelleher I, Keeley H, Corcoran P, Lynch F, Fitzpatrick C, Devlin N, et al. Clinicopathological significance of psychotic experiences in non-psychotic young people: evidence from four population-based studies. Br J Psychiatry 2012;201:26-32, doi:http://dx.doi.org/10.1192/bjp.bp.111.101543.

[7] Wigman JT, van Nierop M, Vollebergh WA, Lieb R, Beesdo-Baum K, Wittchen $\mathrm{HU}$, et al. Evidence that psychotic symptoms are prevalent in disorders of anxiety and depression, impacting on illness onset, risk, and severityimplications for diagnosis and ultra-high risk research. Schizophr Bull 2012;38:247-57, doi:http://dx.doi.org/10.1093/schbul/sbr196.

[8] Armando M, Nelson B, Yung AR, Saba R, Monducci E, Dario C, et al. Psychotic experience subtypes, poor mental health status and help-seeking behaviour in a community sample of young adults. Early Interv Psychiatry 2012;6:300-8, doi:http://dx.doi.org/10.1111/j.1751-7893.2011.00303.x.

[9] Bhavsar V, Maccabe JH, Hatch SL, Hotopf M, Boydell J, Mcguire P. Subclinical psychotic experiences and subsequent contact with mental health services. $\mathrm{Br}$ J Psychiatry Open 2017;3:, doi:http://dx.doi.org/10.1192/bjpo.bp.117.004689.

[10] DeVylder JE, Oh HY, Corcoran CM, Lukens EP. Treatment seeking and unmet need for care among persons reporting psychosis-like experiences. Psychiatr Serv 2014;65:774-80, doi:http://dx.doi.org/10.1176/appi.ps.201300254.

[11] Murphy J, Shevlin M, Houston J, Adamson G. A population based analysis of subclinical psychosis and help-seeking behavior. Schizophr Bull 2012;38:3607, doi:http://dx.doi.org/10.1093/schbul/sbq092.

[12] van der Steen Y, Myin-Germeys I, van Nierop M, Ten Have M, de Graaf R, van Dorsselaer S, et al. "False-positive" self-reported psychotic experiences in the general population: an investigation of outcome, predictive factors and clinical relevance. Epidemiol Psychiatry Sci 2018;(April)1, doi:http://dx.doi.org/ 10.1017/S2045796018000197 [Epub ahead of print].

[13] Werbeloff N, Drukker M, Dohrenwend BP, Levav I, Yoffe R, van Os J, et al. Selfreported attenuated psychotic symptoms as forerunners of severe mental disorders later in life. Arch Gen Psychiatry 2012;69:467-75, doi:http://dx.doi. org/10.1001/archgenpsychiatry.2011.1580.

[14] Bhavsar V, McGuire P, MacCabe J, Oliver D, Fusar-Poli P. A systematic review and meta-analysis of mental health service use in people who report psychotic experiences. Early Interv Psychiatry 2018;12:275-85, doi:http://dx.doi.org/ 10.1111/eip.12464.

[15] Schultze-Lutter F, Michel C, Schmidt SJ, Schimmelmann BG, Maric NP, Salokangas RKR, et al. EPA guidance on the early detection of clinical high risk states of psychoses. Eur Psychiatry 2015;30:405-16, doi:http://dx.doi.org/ 10.1016/j.eurpsy.2015.01.010.

[16] Addington J, Heinssen R. Prediction and prevention of psychosis in youth at clinical high risk. Annu Rev Clin Psychol 2012;8:269-89, doi:http://dx.doi.org/ 10.1146/annurev-clinpsy-032511-143146.

[17] Cotter J, Drake RJ, Bucci S, Firth J, Edge D, Yung AR. What drives poor functioning in the at-risk mental state? A systematic review. Schizophr Res 2014;159(November):267-77, doi:http://dx.doi.org/10.1016/j. schres.2014.09.012.

[18] Brett CMC, Peters ER, McGuire PK. Which psychotic experiences are associated with a need for clinical care? Eur Psychiatry 2015;30:648-54, doi:http://dx. doi.org/10.1016/j.eurpsy.2014.12.005.

[19] Lindgren M, Manninen M, Kalska H, Mustonen U, Laajasalo T, Moilanen K, et al. Predicting psychosis in a general adolescent psychiatric sample. Schizophr Res 2014;158(September):1-6.

[20] Loewy RL, Bearden CE, Johnson JK, Raine A, Cannon TD. The prodromal questionnaire $(\mathrm{PQ})$ : preliminary validation of a self-report screening measure for prodromal and psychotic syndromes. Schizophr Res 2005;79:117-25.

[21] Loewy RL, Therman S, Manninen M, Huttunen MO, Cannon TD. Prodromal psychosis screening in adolescent psychiatry clinics. Early Interv Psychiatry 2012;6:69-75, doi:http://dx.doi.org/10.1111/j.1751-7893.2011.00286.x.

[22] Muthén, L.K. \& Muthén BO. 1998-2017. Mplus User's Guide. Eighth Edition. Los Angeles, CA: Muthén \&amp; Muthén.

[23] Haavik L, Joa I, Hatloy K, Stain HJ, Langeveld J. Help seeking for mental health problems in an adolescent population: the effect of gender. J Ment Heal 2017;1-8, doi:http://dx.doi.org/10.1080/09638237.2017.1340630.

[24] Van Rossum I, Dominguez MDG, Lieb R, Wittchen HU, Van Os J. Affective dysregulation and reality distortion: a 10-year prospective study of their association and clinical relevance. Schizophr Bull 2011;37:561-71, doi:http:// dx.doi.org/10.1093/schbul/sbp101.

[25] Fusar-Poli P, Nelson B, Valmaggia L, Yung AR, McGuire PK. Comorbid depressive and anxiety disorders in 509 individuals with an at-risk mental state: impact on psychopathology and transition to psychosis. Schizophr Bull 2014;40(January):120-31, doi:http://dx.doi.org/10.1093/schbul/sbs136.

[26] Krabbendam L, Myin-Germeys I, Hanssen M, de Graaf R, Vollebergh W, Bak M, et al. Development of depressed mood predicts onset of psychotic disorder in individuals who report hallucinatory experiences. Br J Clin Psychol 2005;44:113-25, doi:http://dx.doi.org/10.1348/014466504X19767.

[27] Perlis RH, Uher R, Ostacher M, Goldberg JF, Trivedi MH, Rush AJ, et al. Association between bipolar spectrum features and treatment outcomes in outpatients with major depressive disorder. Arch Gen Psychiatry 2011;68:351-60, doi:http://dx.doi.org/10.1001/archgenpsychiatry.2010.179.

[28] Wigman JTW, Devlin N, Kelleher I, Murtagh A, Harley M, Kehoe A, et al. Psychotic symptoms, functioning and coping in adolescents with mental illness. BMC Psychiatry 2014;14:, doi:http://dx.doi.org/10.1186/1471-244X-1497.

[29] Gaudiano BA, Zimmerman M. Prevalence of attenuated psychotic symptoms and their relationship with DSM-IV diagnoses in a general psychiatric outpatient clinic. J Clin Psychiatry 2013;74(149), doi:http://dx.doi.org/ 10.4088/JCP.12m07788; 10.4088.

[30] Calkins ME, Moore TM, Satterthwaite TD, Wolf DH, Turetsky BI, Roalf DR, et al. Persistence of psychosis spectrum symptoms in the Philadelphia Neurodevelopmental Cohort: a prospective two-year follow-up. World Psychiatry 2017;16:62-76, doi:http://dx.doi.org/10.1002/wps.20386.

[31] Stochl J, Khandaker GM, Lewis G, Perez J, Goodyer IM, Zammit S, et al. Mood, anxiety and psychotic phenomena measure a common psychopathological factor. Psychol Med 2015;45:1483-93, doi:http://dx.doi.org/10.1017/ S003329171400261X.

[32] Fusar-Poli P, Borgwardt S. Integrating the negative psychotic symptoms in the high risk criteria for the prediction of psychosis. Med Hypotheses 2007;69:959-60, doi:http://dx.doi.org/10.1016/j.mehy.2006.12.044.

[33] Ventura J, Nuechterlein KH, Green MF, Horan WP, Subotnik KL, Mintz J. The timing of negative symptom exacerbations in relationship to positive symptom exacerbations in the early course of schizophrenia. Schizophr Res 2004;69:333-42, doi:http://dx.doi.org/10.1016/S0920-9964(03)00096-3.

[34] Wigman JT, Lin A, Vollebergh WA, van Os J, Raaijmakers QA, Nelson B, et al. Subclinical psychosis and depression: co-occurring phenomena that do not predict each other over time. Schizophr Res 2011;130:277-81, doi:http://dx. doi.org/10.1016/j.schres.2011.03.003.

[35] Van Os J, Linscott RJ. Introduction: the extended psychosis phenotype relationship with schizophrenia and with ultrahigh risk status for psychosis. Schizophr Bull 2012;38:227-30, doi:http://dx.doi.org/10.1093/schbul/sbr188.

[36] Therman S, Lindgren M, Manninen M, Loewy RL, Huttunen MO, Cannon TD, et al. Predicting psychosis and psychiatric hospital care among adolescent psychiatric patients with the prodromal questionnaire. Schizophr Res 2014;158:, doi:http://dx.doi.org/10.1016/j.schres.2014.06.031.

[37] Yung AR, Nelson B, Baker K, Buckby JA, Baksheev G, Cosgrave EM. Psychoticlike experiences in a community sample of adolescents: implications for the continuum model of psychosis and prediction of schizophrenia. Aust N Z J Psychiatry 2009;43:118-28, doi:http://dx.doi.org/10.1080/ 00048670802607188. 
[38] Armando M, Nelson B, Yung AR, Ross M, Birchwood M, Girardi P, et al Psychotic-like experiences and correlation with distress and depressive symptoms in a community sample of adolescents and young adults. Schizophr Res 2010;119:258-65, doi:http://dx.doi.org/10.1016/j.schres.2010.03.001.

[39] Demmin DL, DeVylder JE, Hilimire MR. Screening for sub-threshold psychotic experiences and perceived need for psychological services. Early Interv Psychiatry 2017;11:139-46, doi:http://dx.doi.org/10.1111/eip.12222.

[40] Peters E, Ward T, Jackson M, Morgan C, Charalambides M, McGuire P, et al. Clinical, socio-demographic and psychological characteristics in individuals with persistent psychotic experiences with and without a "need for care. World Psychiatry 2016;15:41-52, doi:http://dx.doi.org/10.1002/wps.20301.

[41] Yung AR, Buckby JA, Cotton SM, Cosgrave EM, Killackey EJ, Stanford C, et al. Psychotic-like experiences in nonpsychotic help-seekers: associations with distress, depression, and disability. Schizophr Bull 2006;32:352-9, doi:http:// dx.doi.org/10.1093/schbul/sbj018.

[42] Bak M, Myin-Germeys I, Delespaul P, Vollebergh W, De Graaf R, Van Os J. Do different psychotic experiences differentially predict need for care in the general population? Compr Psychiatry 2005;46:192-9, doi:http://dx.doi.org/ 10.1016/j.comppsych.2004.08.003.

[43] Rietdijk J, Fokkema M, Stahl D, Valmaggia L, Ising HK, Dragt S, et al. The distribution of self-reported psychotic-like experiences in non-psychotic help- seeking mental health patients in the general population; a factor mixture analysis. Soc Psychiatry Psychiatric Epidemiol 2014;49:349-58, doi:http://dx. doi.org/10.1007/s00127-013-0772-1.

[44] Rössler W, Hengartner MP, Ajdacic-Gross V, Haker H, Gamma A, Angst J. Subclinical psychosis symptoms in young adults are risk factors for subsequent common mental disorders. Schizophr Res 2011;131:18-23, doi:http://dx.doi. org/10.1016/j.schres.2011.06.019.

[45] Breetvelt EJ, Boks MPM, Numans ME, Selten JP, Sommer IEC, Grobbee DE, et al. Schizophrenia risk factors constitute general risk factors for psychiatric symptoms in the population. Schizophr Res 2010;120:184-90, doi:http://dx. doi.org/10.1016/j.schres.2010.03.033.

[46] Kobayashi H, Nemoto T, Murakami M, Kashima H, Mizuno M. Lack of association between psychosis-like experiences and seeking help from professionals: a case-controlled study. Schizophr Res 2011;132:208-12, doi: http://dx.doi.org/10.1016/j.schres.2011.07.029.

[47] Savill M, D’Ambrosio J, Cannon TD, Loewy RL. Psychosis risk screening in different populations using the Prodromal Questionnaire: a systematic review. Early Interv Psychiatry 2017;1-12, doi:http://dx.doi.org/10.1111/eip.12446.

[48] McGorry PD, Nelson B, Amminger GP, Bechdolf A, Francey SM, Berger G, et al. Intervention in individuals at ultra-high risk for psychosis: a review and future directions. J Clin Psychiatry 2009;70(September (9)):1206-12. 